\title{
Terahertz Time-Domain Spectrometer with Precision Delay Line Encoder
}

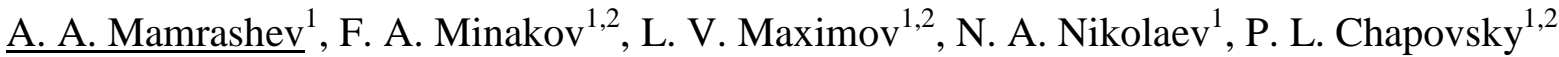 \\ ${ }^{1}$ Institute of Automation and Electrometry SB RAS, Novosibirsk, Russia, mamrashev@iae.nsk.su \\ ${ }^{2}$ Novosibirsk State University, Novosibirsk, Russia
}

\section{Introduction}

Accuracy of optical delay line is crucial for terahertz time-domain spectroscopy (THz-TDS). Typically such delay line consists of a motorized translation stage with a mounted retroreflector. Random and systematic errors in positioning of the translation stage result in distortion of sampled terahertz pulses and hence terahertz spectra.

It is intuitive that random misplacements in the sampling position (registration jitter) translate into an error in the amplitude of the measured terahertz signal proportional to its derivative. This results in a higher noise floor in the terahertz spectra [1,2]. It was recently found that periodic sampling errors lead to creation of spurious mirror spectra around the error's frequency [3]. In a typical terahertz measurement a series of sampled terahertz pulses or spectra are averaged to achieve better signal to noise ratio. However, even small delays in the sampling start times can actually increase the noise level in the averaged signal [4]. So it was suggested to align terahertz pulses in the time domain before averaging using algorithms from [5].

In the custom-made THz-TDS developed at IA\&E SB RAS [6] we used motorized translation stage 8MT173-50-20 (Standa Ltd, Lithuania) with the full movement range of $50 \mathrm{~mm}$ (time range of $333 \mathrm{ps}$ ) and the step of $1.25 \mu \mathrm{m}$ (time step of $8.34 \mathrm{fs}$ ). In order to independently measure translation stage position in this study we installed an optical encoder Resolute RL32BAT001B50 with RTLA absolute scale (Renishaw, UK) (see Fig. 1). The scale length was $100 \mathrm{~mm}$ and its accuracy was $0.5 \mu \mathrm{m}$. The encoder resolution was $1 \mathrm{~nm}$. Thus we detected delay line inaccuracies and corrected them.

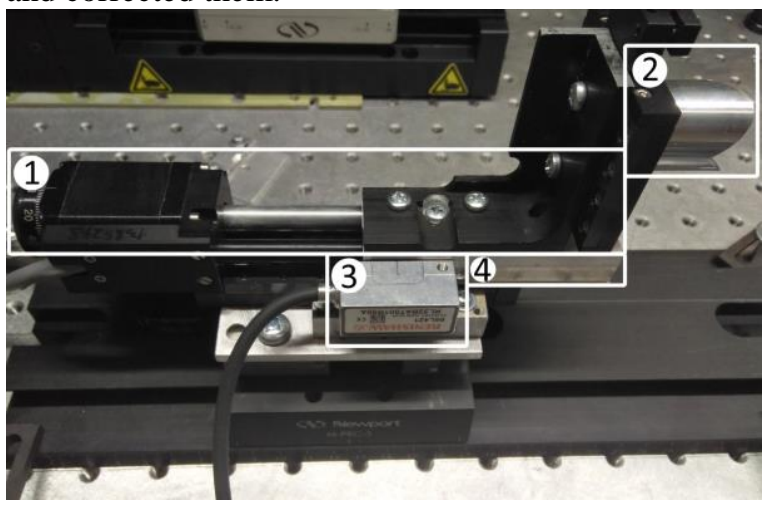

Fig. 1. Upgraded optical delay line consisting of the motorized translation stage (1), the retroreflector (2), the optical encoder (3) and the high accuracy scale (4)

\section{Results}

We compared position of the translation stage $\left(\mathrm{L}_{\mathrm{st}}\right)$ with the position measured by the optical encoder $\left(\mathrm{L}_{\mathrm{en}}\right)$. We studied their difference for four transla- tion stages by installing the encoder on each (Fig. 2). It was found that three systematic errors are associated with the measurements: growing offset of the position, periodic error, and drift of the starting position.

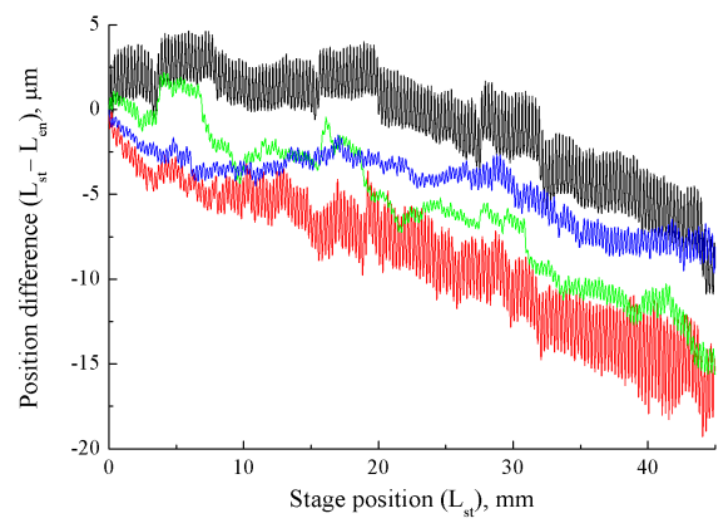

Fig. 2. Difference between the positions of the translation stage $\left(\mathrm{L}_{\mathrm{st}}\right)$ and the optical encoder measurements $\left(\mathrm{L}_{\mathrm{en}}\right)$. The data are presented for four different stages

First, it can be seen from the Fig. 2 that all four translation stages exhibit growing position offset that can roughly be approximated by a linear function. Abrupt changes of the offset may be explained by mechanical defects in the translation stage screw. Total offset varies from -8 to $-15 \mu \mathrm{m}$ for different stages. Such offset manifests itself in a compressed terahertz pulse and consequently stretched terahertz spectrum, i. e. having linearly increasing frequency shift. In our experiments it leads to a total shift of up to $400 \mathrm{MHz}$ at $1 \mathrm{THz}$. Correction of such systematic error is crucial in the measurements of narrow-band absorption peaks such as spectra of nuclear spin isomers of water vapor [7].

Second, we notice periodic sinusoidal sampling error that becomes apparent after zooming in on the finer structure of the difference between translation stage position and encoder measurements (see Fig. 3).

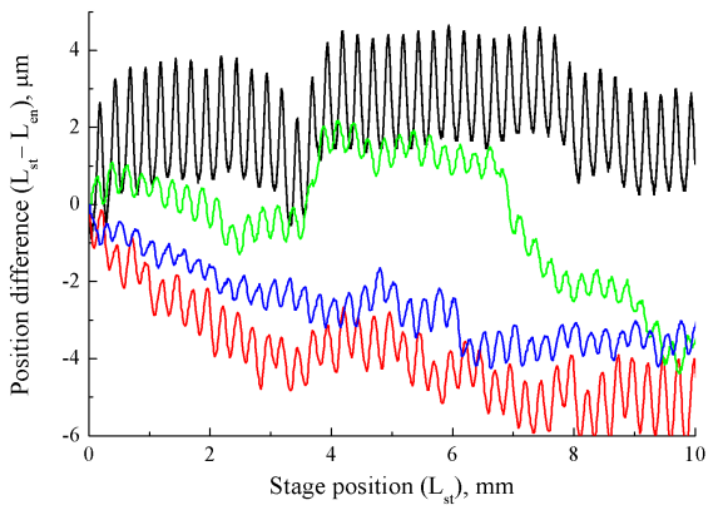

Fig. 3. Difference between the positions of the translation stage $\left(\mathrm{L}_{\mathrm{st}}\right)$ and the optical encoder measurements $\left(\mathrm{L}_{\mathrm{en}}\right)$. The data are presented for four different stages 
The period of the oscillations is $250 \mu \mathrm{m}$ that corresponds well with a single revolution of the stage screw. The amplitude of the oscillation varies from 0.3 to $1.6 \mu \mathrm{m}$ for different stages. According to [3] such periodic sampling errors result in the presence of additive spurious mirror copies of the main pulse spectrum around error's frequency and its harmonics. In our case oscillation period of $250 \mu \mathrm{m}$ corresponds to the frequency of $600 \mathrm{GHz}$. The amplitude of the spurious spectra is proportional to the amplitude of the oscillations. In our measurements it results in the proportionality coefficient as low as $10^{-3}$ which is comparable with the signal-to-noise ratio making this error difficult to detect.

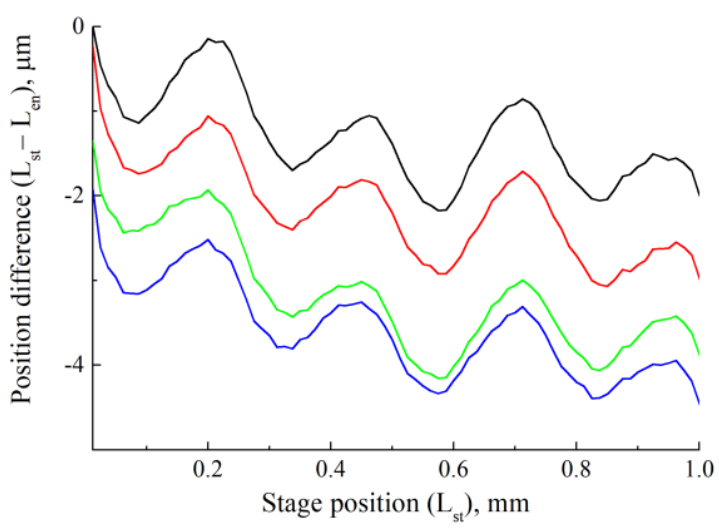

Fig. 3. Difference between the positions of the translation stage $\left(\mathrm{L}_{\mathrm{st}}\right)$ and the optical encoder measurements $\left(\mathrm{L}_{\mathrm{en}}\right)$. The data are presented for four consecutive measurements of the same translation stage

Finally, we find that the starting position of the same translation stage in the consecutive measurements drifts by less than $1 \mu \mathrm{m}$ and the drift magnitude gets smaller each time. It results in time delays of the measured terahertz pulses or equivalently in phase shifts in their spectra. This error requires correction before averaging of the series of consecutive terahertz measurements. Otherwise, it may lead to an increased error in the averaged signal.
In conclusion, we found several systematic errors in the optical delay line of THz-TDS by independently measuring its absolute position with precision optical encoder. We found that correction of the position offset of the delay line is crucial in gas analysis. Correction of the starting position drift should be applied before averaging the series of terahertz pulses measured consecutively. Periodic sampling error resulted in a small additive spectral error which can only be found in certain spectra such as ones provided by high contrast band pass filters.

\section{Acknowledgement}

The study was financially supported by the Russian Science Foundation (RSF), project 17-12-01418.

\section{References}

1. Jahn D. et al. On the influence of delay line uncertainty in $\mathrm{THz}$ time-domain spectroscopy // Journal of Infrared, Millimeter, and Terahertz Waves. 2016. Vol. 37, No. 6. P. 605-613.

2. Cohen N. et al. Experimental signature of registration noise in pulsed terahertz systems // Fluctuation and Noise Letters. 2006. Vol. 6, No. 1. P. L77-L84.

3. Rehn A. et al. Periodic sampling errors in terahertz time-domain measurements // Optics Express. 2017. Vol. 25, No. 6. P. 6712.

4. Humphreys D.A., Naftaly M., Molloy J.F. Effect of time-delay errors on $\mathrm{THz}$ spectroscopy dynamic range // 39th International Conference on Infrared, Millimeter, and Terahertz Waves (IRMMW-THz 2014). IEEE, 2014.

5. Coakley K.J., Hale P. Alignment of noisy signals // IEEE Transactions on Instrumentation and Measurement. 2001. Vol. 50, No. 1. P. 141-149.

6. Antsygin V.D. et al. Effect of a Magnetic Field on Wideband Terahertz Generation on the Surface of Semiconductors // IEEE Trans. Terahertz Sci. Technol. 2015. Vol. 5, No. 4. P. 673-679.

7. Mamrashev A.A. et al. Detection of Nuclear Spin Isomers of Water Molecules by Terahertz Time-Domain Spectroscopy // IEEE Transactions on Terahertz Science and Technology. 2018. Vol. 8, No. 1. P. 13-18. 